\title{
New Media Marketing Strategy Optimization in the Catering Industry Based on Deep Machine Learning Algorithms
}

\author{
Zikang Peng $(\mathbb{D}$ \\ School of Marxism, Henan Finance University, Zhengzhou 450046, Henan, China \\ Correspondence should be addressed to Zikang Peng; pengzikang@hafu.edu.cn
}

Received 4 December 2021; Revised 30 December 2021; Accepted 13 January 2022; Published 4 February 2022

Academic Editor: Miaochao Chen

Copyright (c) 2022 Zikang Peng. This is an open access article distributed under the Creative Commons Attribution License, which permits unrestricted use, distribution, and reproduction in any medium, provided the original work is properly cited.

\begin{abstract}
With the in-depth development of new-generation network technologies such as the Internet, big data, and cloud intelligence, people can obtain massive amounts of information on mobile phones or mobile platforms. The era of unreachable big data has arrived, which raises questions for the development of corporate marketing. With the development of Internet technology, people use mobile terminals for longer and longer periods of time. New media has gradually become the mainstream of the media arena. It has distinctive features such as freedom to find audiences, diverse content forms, and timeliness of information release, which has changed the traditional. The marketing model has a profound impact on the development of the market. This article uses relevant theories, such as new media, marketing, and catering industry marketing strategies, studies the related concepts and characteristics of new media, clarifies the impact of the development of new media on the catering industry and audience groups, and studies the impact of the catering industry from multiple dimensions. Based on the development factors in the new media environment, combined with marketing theory, it puts forward suggestions for catering companies to use new media to carry out marketing planning in product innovation, improving information channels, creating network events and topics, and promoting innovation and health in the catering industry. And a marketing strategy is proposed based on deep machine learning algorithms; including a cloud server, the cloud server communicates with the e-commerce software platform and the input of physical sales is recorded. The adopted cloud server is connected with data collection, data processing, and communication module. The communication module is connected with a deep machine learning algorithm system; that is, deep machine learning algorithm system is connected with a sales platform in communication. The sales platform is connected with advertising settings and advertising, and the advertising is electrically connected with an algorithm of advertising delivery methods. Advertisement delivery method algorithm communication is connected to the cloud server. This article uses deep machine learning algorithms to process the data information to make the data information easy to view and clear. The advertisement delivery method algorithm calculates the best way of advertising and then calculates the advertisement to deliver.
\end{abstract}

\section{Introduction}

Marketing refers to the process by which a company discovers or explores the needs of prospective consumers and lets consumers understand the product and then purchase the product. Classic business management courses such as MBA and EMBA all regard marketing as an important module for management and education of managers. Marketing is an activity, process, and system that brings economic value to customers, partners, and the entire society in creating, communicating, disseminating, and exchanging products $[1,2]$. It mainly refers to the process in which marketing conducts business activities and sales behaviors for the market at the same time, that is, the process of transforming business and sales. Marketing strategy is that the company takes customer needs as the starting point and obtains information on customer demand and purchasing power based on experience, as well as business expectations. Organize various business activities in a planned way. It is a series of measurable and controllable activities aimed at improving sales and manufacturer's reputation for a certain target market. It is a combination of multiple marketing methods such as products, prices, channels, promotions, and public 
relations strategies. Various marketing strategies still have various problems [3-5].

This has brought forward transformation and innovation to the marketing development of enterprises such as requirements for development. The effect of the traditional marketing model is diminishing, and the new media interaction model has gradually become a rising star on the market stage. Various APPs developed in conjunction with smart phones have emerged, and they have innovated marketing models. Traditional enterprises have gradually begun to innovate marketing methods in new dimensions. So, what we see is that new media platforms such as various apps, WeChat, Weibo, and Douyin continue to push all kinds of information to consumers. In order to keep up with market development, major companies have launched their own official channels on new media platforms to innovate information dissemination and marketing methods. The BP sketch is shown in Figure 1.

For traditional catering companies, in the face of this era of "fragrant wine fears no dark alleys," they must also take advantage of the power of new media platforms to be better The times have developed even greater.

Researching the development of mainstream catering operations, almost all brand catering companies will build their own APP or official channels based on new media platforms to release or push information. This just shows that the catering industry of the catering industry audience has begun to be affected by the new media. Enterprises reexamine and think about the point of force and development direction: paying attention to the quality of dishes and paying attention to service are only the basis for the development of the industry. The use of new media platforms to carry out marketing planning has become more and more important. In the catering industry, whether it is Internet catering brands, traditional catering brands, or Chinese time-honored brands, they have gradually realized the importance of new media marketing. It is an inevitable choice to compete with peers and stand out in fierce competition [6,7].

However, with the development of network technology, especially the mobile terminal network, people can obtain massive amounts of information on mobile phones or mobile terminal platforms. The era of unreachable big data has arrived. Marketing management has gone through 50 years of research and development. Domestic and foreign scholars have become more and more perfect in marketing management and new media marketing and continue to enrich their basic theories. In terms of researching new media and its marketing concepts, today's industry and academia have not reached a consensus. Different researchers have different research results and focuses.

$\mathrm{Yu}$ Guoming pointed out that the characteristics of new media include fragmentation, sharing, and digitization. Jingdong and Su Baohua relied on the definition of "categorical plus species difference" to explore the important position of enterprises in constructing new media marketing and selected the more representative microblogs in new media as the target during the research. Concepts, products, culture, and so on are delivered to more and more consumers through the Internet platform [8].

Liu Ning and Chen Lei analyzed the current growth of new media marketing, and with the help of SWOT analysis methods, they analyzed the external opportunities and tests encountered in the current growth of new media marketing, internal advantages and disadvantages, and mentioned opinions: first, improve the environment in which the online market is located and build a good competition system; second, improve the industry's service system. From sales to after-sales service, a responsible person system needs to be established, and major responsibility issues should be adopted, the method of accountability; the third is to improve the growth and implementation of network marketing to achieve a new situation where the network platform and the previous catering industry can achieve a win-win, share, and common growth [9-14].

Gary S. Lynn analyzed the growth path of companies of different scales in the new media environment. After research, it was found that large companies with sufficient funds can support the company's new media marketing strategy, and small companies can also use new media. The feature of low cost promotes part of the marketing work, which means that companies with different scales and marketing companies in a different new media environment can get opportunities in the process of growth.

Jay M. Bernhardt and Darren Mays elaborated on the current existing new media platforms and mobile communication technologies. The research gave the view that new media marketing platforms can rely on many channels of communication to stimulate consumers' hidden thinking, and further transform the previous commercial marketing methods. Relevant marketing staff need to explore the influence of new media in their past marketing work and use the new media to explore the true needs of consumers $[15,16]$.

After investigation, Ding Yuping came to the conclusion that consumers' catering requirements no longer rest on their stomachs. They are increasingly pursuing specialization and diversification in their catering needs. The catering services should be optimized according to the changes in consumer needs; otherwise, they will be cruel. There is bound to be no place in market competition. It is necessary to understand that only by seizing consumers can we gain a firm foothold in the market. The catering industry needs to be highly sensitive to the market, and whether it is food conception or service, it needs to be based on consumer experience $[17,18]$.

Du Hongyan studies the continuous integration of services in the catering industry. People's focus is not only on service, but also on the time of serving, the price and taste of the dishes, and so on, which will affect customer experience. The development of catering companies must pay attention to consumers. Feeling, only by exploring, and feeling marketing methods can make customers become repeat customers again. Therefore, catering companies should try to explore various experiential marketing methods in the catering industry and promote and adjust the 


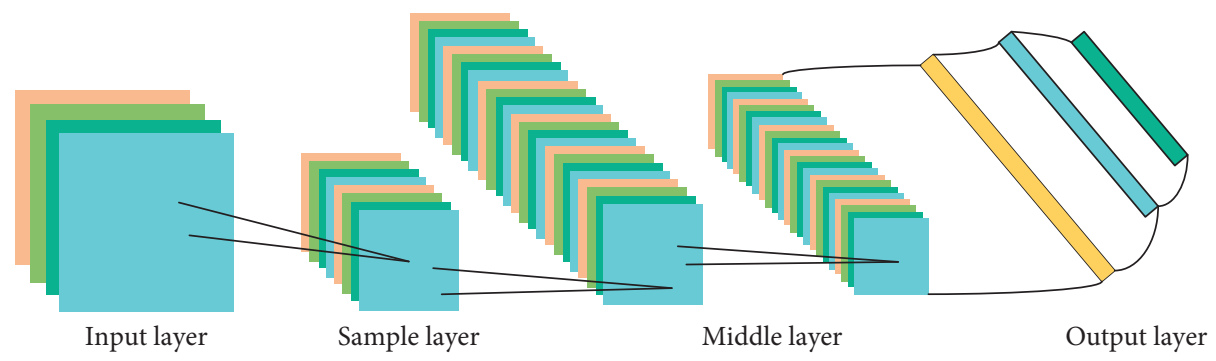

FIGURE 1: BP method sketch.

long-term healthy growth of the catering industry through marketing thinking [19-23].

The first historical marketing information obtains the first dimension information of the first merchant; obtains the first degree of influence of the first dimension on the first historical marketing effect; and determines whether the first degree of influence exceeds a first preset threshold. When the first degree of influence exceeds the first preset threshold, it is determined to compose the first dimensional information into a first marketing strategy template; according to the first marketing task of the first merchant and the first marketing, the strategy template obtains the first optimal marketing strategy and sends the first optimal marketing strategy to the first user, thereby solving the problem that the merchants in the prior art cannot control the impact of marketing strategies on marketing effects in real time and cannot effectively and timely optimize the technical problems of marketing strategy and low product conversion rate, achieving timely control of the impact of marketing strategy on marketing effect, continuously optimizing marketing strategy with marketing effect, increasing output value, increasing product conversion rate, and reducing marketing cost. However, it does not solve the problems of the single acquisition method of data resources in the existing marketing strategy, the acquisition of fewer resources, and the inability to achieve deep learning processing of data resources, and the setting and placement of advertisements based on data resources. For this reason, we propose a marketing strategy based on deep machine learning algorithms [24-28].

The concept of deep learning model needs to be traced back to 2006. Geoffrey Hinton used neural network to complete the dimensionality reduction of data and published the results in "Science." Since then, the concept of deep learning has been continuously extended to other fields and has been successfully used. For example, three leading figures in the field of deep learning, Yann LeCun, Yoshua Bengio, and Geoffrey Hinton published a comprehensive research article titled "Deep Learning" in the journal Nature in 2015, in which they launched a detailed study of deep learning. Discussion. In general, the main content of deep learning is that it mainly learns various feature expressions through a model composed of multiple cascaded network layers, and it also has the characteristics of multiple abstract levels. In addition, it also needs to use the backpropagation algorithm to guide the machine to self-learn by changing the internal variables and explore the deeper content contained in the data sample. In fact, this method of using backpropagation or hierarchical models to expand corresponding learning has been used in media such as images, videos, text, and audio. For now, the more successful training network types are Deep Belief Networks based on DBN algorithm, Generative Adversarial Networks based on model optimization training, Long Short-Term Memory to solve RNN, feedforward neural network (English name is Convolutional Neural Networks), and so on. In addition, many scholars have paid attention to the backpropagation algorithm of training the network. Therefore, people began to develop more efficient algorithms, including AdaDelta, Adam, and RMSprop [29-32].

Therefore, this paper uses deep machine learning algorithms to process the data information to make the data information easy to view and clear. The advertisement delivery method algorithm calculates the best way of advertising and then calculates the advertisement to deliver.

\section{Deep Learning}

Artificial intelligence is considered to be a "knowledge-related subject" due to its comprehensiveness of disciplines, and due to its integration of high-precision technology, it is considered to be able to be used to realize intelligent activities that replace humans with machines, which also represents relevantly the center and core idea of the field. That is, through the frame-by-frame study of human motion trajectories, many commands are given to the machine, and the machine's own knowledge system is even cultivated to replace humans in handling some behaviors/businesses. The research content is mainly to control the machine through code to imitate and learn the characteristics of humans. The act of consciousness conducts learning and knowledge theory and operation (see Figure 2). The success of deep learning methods is inseparable from the following three key factors: (1) large-scale labeled training data sets; (2) convolutional neural networks have unique advantages in feature expression; and (3) powerful computing capabilities.

In addition, there are also documents that propose a method and device for optimizing marketing strategies based on marketing effects. Although it has achieved the first business information of the first business, the first business information includes the first historical marketing information. As the core of artificial intelligence disciplines, machine learning is the basis for making computers intelligent. It has interoperability and tolerance of disciplines and 


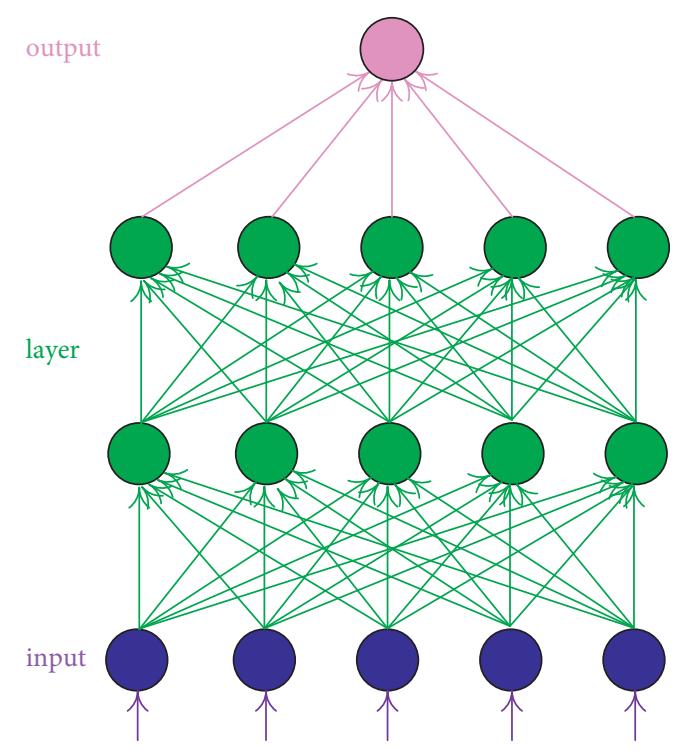

Figure 2: Traditional neural network.

can organically integrate multiple types of theories. The main content is to use algorithms to enable the machine to have a certain simulation ability, to independently complete some behaviors that only humans can do before, to establish its own knowledge structure and skill system, and to update and improve its knowledge structure in real time. As one of the most important technologies of artificial intelligence, it is the basis for the realization of mechanical intelligence. Generally speaking, machine learning is mainly used to summarize and integrate information, which is then deduced by artificial intelligence.

Deep learning is the core algorithm in machine learning. This definition first appeared in 2006. Researchers such as Geoffrey proposed a technique to reduce the dimensionality of data with the help of neural networks and published this result in the Science journal. This concept is one of the most important advances in artificial intelligence technology in the past decade. It has made great breakthroughs in many technologies, including image and video analysis, audio discrimination, natural language processing, computer hearing, and multimedia. In the current use of neural network models, it teaches computers to think and understand the world in a human way. In 1940, the concept of neural networks began to appear, which is a study of the formation mechanism of brain consciousness and simulations to answer various types of machine learning questions. In 1986, Rumelhart et al. discovered that the backpropagation algorithm can be used in the process of training neural networks. This famous discovery was published in the Nature journal and has been widely used today.

At present, convolutional neural networks can be applied in the field of image recognition with the help of deep learning algorithms, but the collection of large-scale labeled data sets is still a difficult point. Deep learning models are mainly composed of deep neural network models. These models usually have relatively complex network structures and a large number of parameters. They often need to rely on a large amount of artificially labeled data for training to obtain good performance in a data-driven manner; for example, ImageNet, an important database in the field of computer vision, has more than 10 million pictures.

Normal deep learning model training process. Normal data analysts will write data processing code and model training code based on desensitized sample data in the debugging environment of catering data. After the code is debugged, the data analyst will send it to the operating environment of the catering data. In the operating environment, the data processing code will run on the full amount of training data $D$, and the processed training data $D^{\prime}$ will be obtained, which is defined as follows:

$$
D^{\prime}=\left\{\left(x_{i}, y_{i}\right)\right\}_{i=1}^{n}, \quad 1 \leq i \leq n(1) .
$$

Among them, $x_{i}$ and $y_{i}$ are the feature value and label value of each piece of data, respectively. The optimization process of the model is shown in the following formula:

$$
\min _{\theta} \sum_{i=1}^{n} L\left(y_{i}, f_{\theta}\left(x_{i}\right)\right)+\phi \text {. }
$$

Among them, the function $L$ is the loss function of deep learning, which is used to evaluate the difference between the judgment result of the function $f_{\theta}$ on $x_{i}$ and the real label value $y_{i} . \Phi$ is a regular term, usually used to prevent the AI model from overfitting.

The data theft attack method mainly acts on the data processing stage. The attacker maliciously modifies the data enhancement function in the data processing process. This article defines the malicious data DM as shown in the following equation:

$$
D_{M}=\left\{\left(x_{i}^{M}, y_{j}^{M}\right)\right\}_{j=1}^{M}, \quad 1 \leq j \leq m
$$

For the attack in the model training phase, first extract the real number string $s$ from the feature values of the 
training data. The number of elements in the string is the same as the number of parameters in the model parameter $\theta$, which is $k$. In the training process, the attacker modifies the regular term as shown in the following equation:

$$
\Omega_{1}(\theta, s)=-\alpha \frac{\left|\sum_{i=1}^{k}\left(\theta_{i}-\bar{\theta}\right)\left(s_{i}-\bar{s}\right)\right|}{\sqrt{\sum_{i=1}^{k}\left(\theta_{i}-\bar{\theta}\right)^{2}} \sqrt{\sum_{i=1}^{k}\left(s_{i}-\bar{s}\right)^{2}}}
$$

where $\bar{\theta}$ and $\bar{s}$ refer to the average value of all elements in $\theta$ and $s$, and the parameter $a$ controls the influence of the malicious regular term on the training process.

Another attack against the model training phase is

$$
\Omega_{2}(\theta, s)=-\alpha \frac{\sum_{i=1}^{k}\left|\max \left(0,-\theta_{i} s_{i}\right)\right|}{k} .
$$

In the normal training process, the regular term of the deep learning loss function generally selects the L1 paradigm or the L2 paradigm, as shown in the following equations:

$$
\begin{array}{r}
\phi(\theta)=\lambda \sum_{i}\left|\theta_{i}\right|, \\
\phi(\theta)=\lambda \sum_{i} \theta_{i}^{2} .
\end{array}
$$

\section{Marketing Strategy Based on Deep Learning}

Generally speaking, data collection and labeling are usually done manually, and obtaining large-scale, high-quality data sets can be time-consuming and expensive. In addition, some data is not easy to obtain. The structure diagram of the marketing strategy system based on deep learning proposed in this paper is shown in Figure 3. It includes a cloud server; the cloud server is in communication connection with the e-commerce software platform, the cloud server also communicatively receives the input of physical sales records, the cloud server is communicatively connected to data collection, and the data collection is electrically connected to data processing, The data processing is electrically connected to a communication module, the communication module is communicatively connected to a deep machine learning algorithm system, the deep machine learning algorithm system is communicatively connected to a sales platform, and the sales platform is communicatively connected to an advertisement setting. The advertisement setting communication is connected with advertisement placement, the advertisement placement is electrically connected with the advertisement placement algorithm, the advertisement placement algorithm is communicatively connected to the cloud server, and the communication module is communicatively connected to the cloud server and the e-commerce company. The software platform includes customer information, e-commerce browsing records, and e-commerce purchase records. Deep learning models are mainly composed of deep neural network models. These models usually have relatively complex network structures and a large number of parameters.
The data collection receives and collects the data information of the e-commerce software platform and entity sales records through the cloud server and transmits it to the data processing. After the data processing is completed, the data is processed through the communication module. The data information is transmitted to the deep machine learning algorithm system, and the deep machine learning algorithm system transmits the data information to the sales platform after calculating and processing the data. The advertisement is set and delivered, and then the advertisement content is sent to the cloud server through the advertisement delivery method algorithm, and the cloud server delivers the advertisement content (see Figures 4 and 5). They often need to rely on a large amount of artificially labeled data for training to obtain good performance in a data-driven manner; for example, ImageNet, an important database in the field of computer vision, has more than 10 million pictures.

In order to achieve interception of the information in the e-commerce software in the hardware device, in this embodiment, preferably, the e-commerce software platform is installed on the hardware device, and the hardware device intercepts the information in the e-commerce software platform. The customer information, the e-commerce browsing record, and the e-commerce purchase record are implemented by embedding an e-commerce software browsing recognition program in the hardware device. For example, in medical research, there are not many individuals with many rare diseases. Even if statistics are performed on a global scale, only about a hundred data samples can be obtained.

In order to realize the interception of the information of the e-commerce software and make the e-commerce software browsing recognition program easy to program and maintain, in this embodiment, preferably, the e-commerce software browsing recognition program uses the FB Pixel program code. The FB Pixel program code is a Java program sequence. The FB Pixel program code is embedded in the hardware device to identify the e-commerce website, the customer information, the e-commerce browsing record, and the e-commerce purchase record. The FB Pixel program code tracks and records these potential consumption behaviors.

In order to facilitate the setting of the interception duration of the e-commerce software browsing identification program, and the advertisement placement within the duration, in this embodiment, it is preferable that the FB Pixel program code can be used to track and record the duration of user visits. Define settings, and within the set time period, the advertisement is delivered to the hardware device through the cloud server, and the customer can increase the familiarity of the advertisement product when viewing the advertisement of the advertisement, as shown in Figures 6 and 7. In this case, how to obtain a large number of data sets and be able to use them? Training with models is the most difficult problem. As higher organisms, humans can use small-scale labeled samples to improve personal cognition; analogous to the field of deep learning, small-shot learning is a research topic that gives convolutional neural networks fast learning characteristics. 


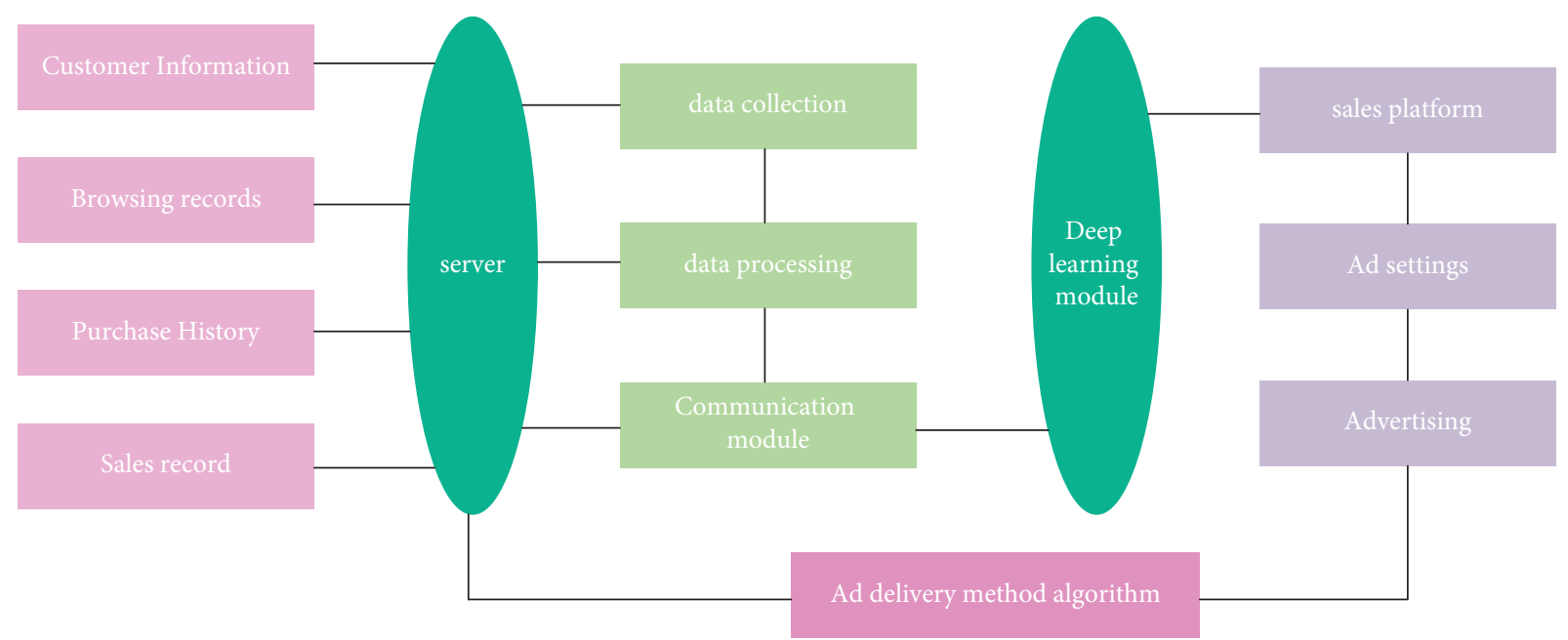

FIGURE 3: Marketing strategy system based on deep learning.

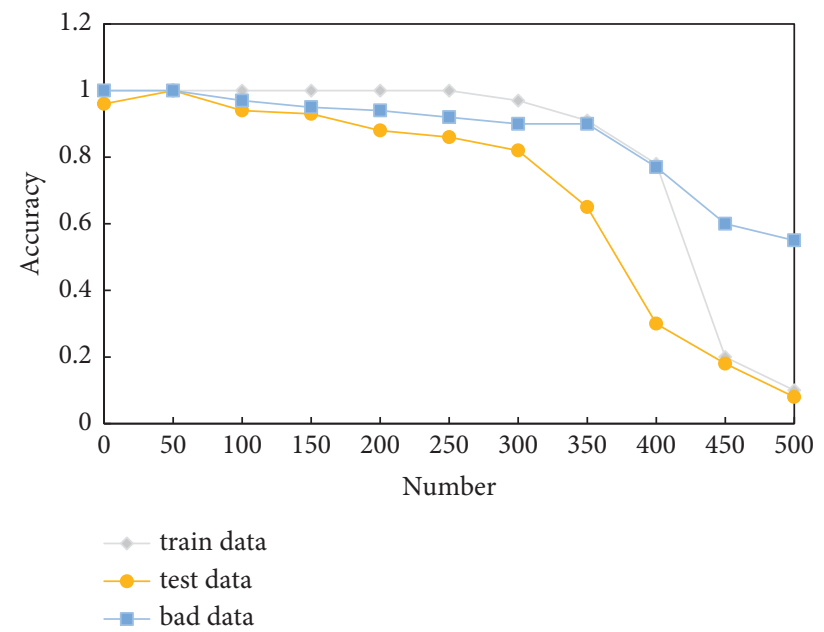

Figure 4: Result comparison.

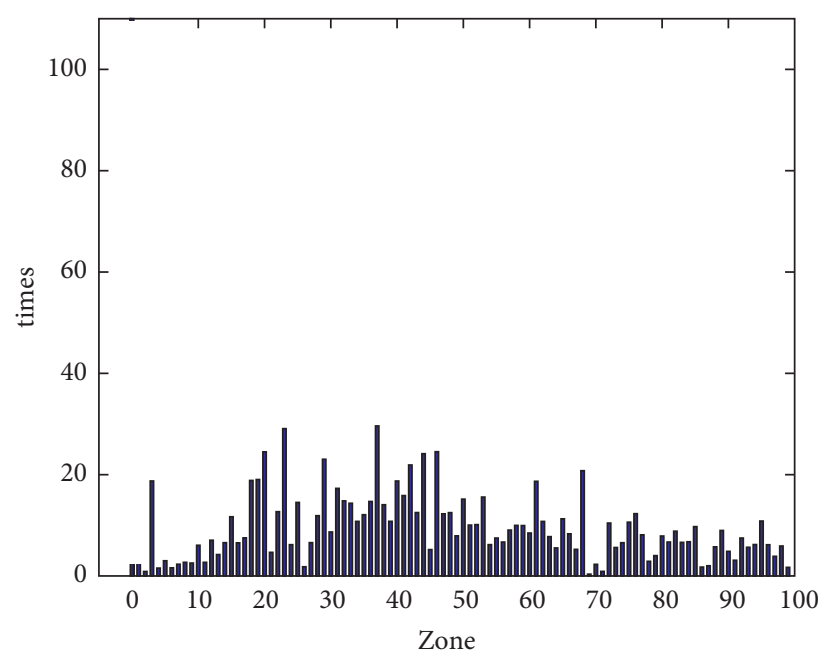

Figure 5: Training results. 


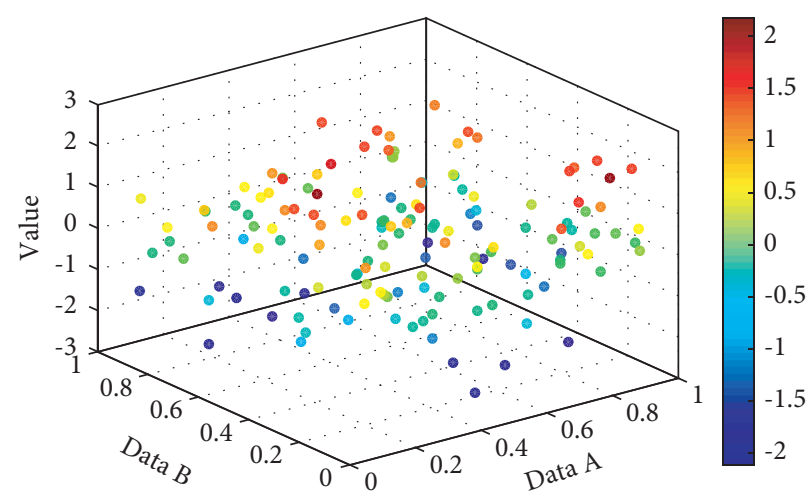

Figure 6: Analysis result.

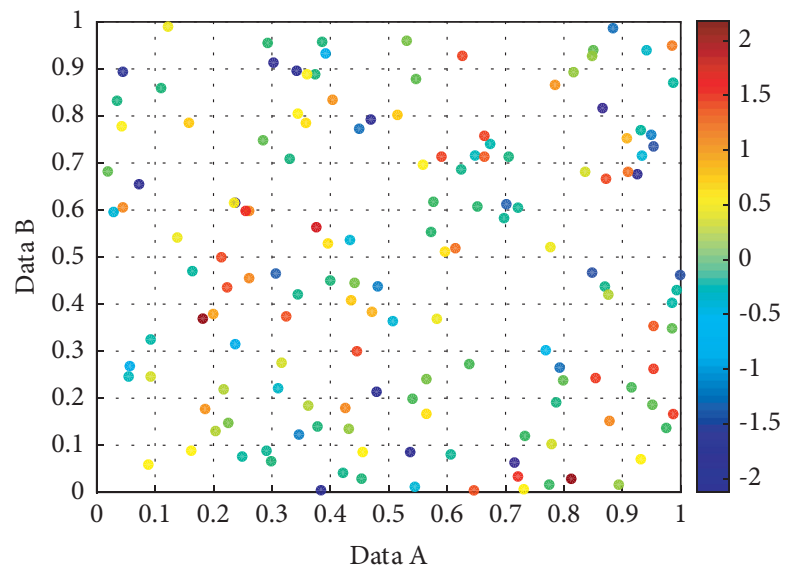

FIgURE 7: Different data results.

In order to realize effective processing, analysis, decoding, and conversion of data information in the cloud server, in this embodiment, preferably, the data processing includes a data receiving module, a data analysis module, a data processing module, a data decoding module, and a conversion module.

In order to achieve algorithmic processing of data in the deep machine learning algorithm system, in this embodiment, preferably, the deep machine learning algorithm system includes backpropagation, stochastic gradient descent, learning rate attenuation, dropout, batch normalization, and long and short-term memory. The merchant purchases records and then transmits the data information to the cloud server, and the physical sales record also transmits the data information to the cloud server, and the cloud server stores the data information in the cloud database, and then the data collection will be collected from the cloud server.

In order to realize the calculation processing through the batch standardization algorithm, in this embodiment, preferably, the calculation formula of the batch standardization is as follows:

Input: values of $x$ over a mini-batch are

$$
\beta=\left\{x_{1}, \ldots, m\right\},
$$

Parameters to be learned: $\gamma, \beta$.
Then, the output can be derived by the following equations:

$$
\begin{aligned}
& \mu_{\beta} \leftarrow \frac{1}{m} \sum_{i=1}^{m} x_{i}, \\
& \sigma_{\beta}^{2} \leftarrow \frac{1}{m} \sum_{i=1}^{m}\left(x_{i}-\mu_{\beta}\right)^{2}, \\
& \widehat{x}_{i} \leftarrow \frac{x_{i}-\mu_{\beta}}{\sqrt{\sigma_{\beta}^{2}+\beta}}, \\
& y_{i} \leftarrow \gamma \widehat{x}_{i}+\beta \equiv B N_{\gamma, \beta}\left(x_{i}\right) .
\end{aligned}
$$

In order to realize the setting of advertisement content and the delivery of advertisements, in this method, preferably, the advertisement setting includes image advertisements, dynamic advertisements, video advertisements, audio advertisements, and text advertisements, and the advertisement placement includes browse pages, emails, video time periods, dating software, game intermittent time periods, and physical advertisements.

In order to realize the algorithm calculation of the advertisement delivery method to obtain the best delivery method, in this article, preferably, the advertisement delivery 


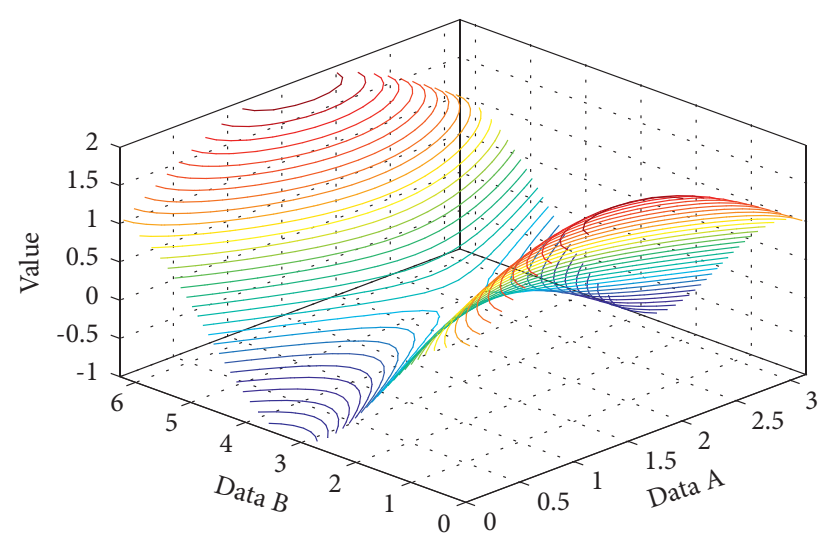

Figure 8: Data comparison.

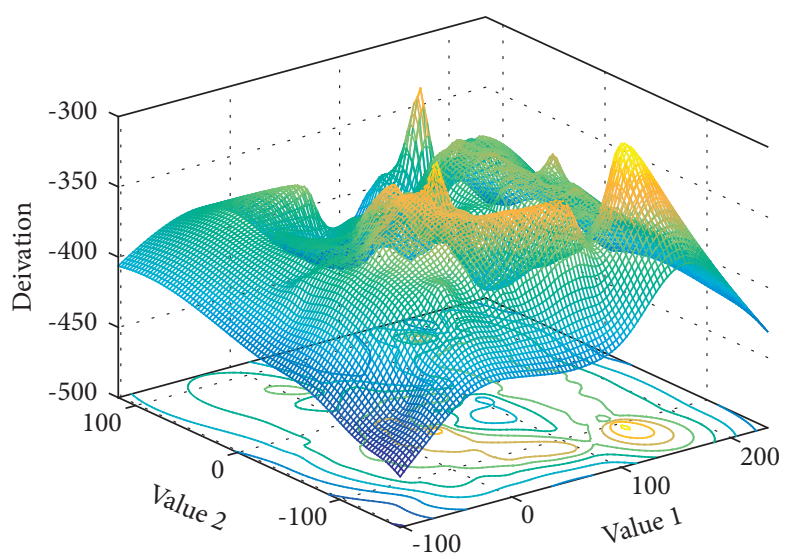

FIGURE 9: Deviation at different values.

method algorithm includes a greedy algorithm, an ant colony algorithm, and a genetic algorithm.

In order to enable the cloud server to store data information and perform classified storage, in this article, preferably, the cloud server includes a cloud database, the cloud database is provided with multiple groups, and one group of the cloud database is used. The e-commerce browsing records are stored, a group of the cloud databases are used to store the e-commerce purchase records, a group of the cloud databases are used to store physical sales records, and a group of the cloud databases are used to store customer information, as shown in Figures 8 and 9. In summary, the working principle and use process of the method proposed in this article can be summarized as follows: when using, the e-commerce software browsing recognition program in the hardware device realizes the extraction of customer information, e-commerce browsing records, and e-commerce software platforms in the e-commerce software platform.

After the data information in the data collection is processed through data processing, it is transmitted to the deep machine learning algorithm system through the communication module for processing, and the deep machine learning algorithm system transmits the data information to the sales platform, and then the sales platform uses advertising settings and the advertising content is set and delivered, and the advertising delivery algorithm is used to calculate the delivery method in the advertising delivery map, and the best delivery method is obtained through calculation, and then the advertisement is delivered through the cloud server to complete marketing.

\section{Conclusions}

The conclusion is summarized as follows:

(1) This paper uses relevant theories such as new media, marketing, and catering industry marketing strategies, studies the related concepts and characteristics of new media, clarifies the impact of the development of new media on the catering industry and audience groups, and studies the impact of the catering industry from multiple dimensions.

(2) Based on the development factors in the new media environment, combined with marketing theory, it puts forward suggestions for catering companies to use new media to carry out marketing planning in product innovation, improve information channels, create network events and topics, and promote innovation and health in the catering industry. Develop. 
(3) A marketing strategy is proposed based on deep machine learning algorithms; including a cloud server, the cloud server communicates with the e-commerce software platform and the input of physical sales is recorded; the adopted cloud server is connected with data collection, data processing, and communication module.

\section{Data Availability}

The data used to support the findings of this study are available from the corresponding author upon request.

\section{Conflicts of Interest}

The author declares that there are no conflicts of interest.

\section{References}

[1] K. He, X. Zhang, S. Ren, and J. Sun, "Deep residual learning for image recognition," in Proceedings of the IEEE conference on computer vision and pattern recognition, pp. 770-778, Las Vegas, NV, USA, June 2016.

[2] M. Yun, J. Zhao, J. Zhao, X. Weng, and X. Yang, "Impact of invehicle navigation information on lane-change behavior in urban expressway diverge segments," Accident Analysis \& Prevention, vol. 106, no. 1, pp. 53-66, 2017.

[3] S. Kumar Dwivedi, R. Amin, and V. Satyanarayana, "Blockchain-based secured event-information sharing protocol in internet of vehicles for smart cities," Computers \& Electrical Engineering, vol. 86, no. 1, pp. 1-9, 2020.

[4] Z. Khan and S. Amin, "Bottleneck model with heterogeneous information," Transportation Research Part B: Methodological, vol. 112, no. 1, pp. 157-190, 2018.

[5] J. M. Cairney, K. Rajan, and D. Haley, "Mining information from atom probe data," Ultramicroscopy, vol. 159, no. 1, pp. 324-337, 2020.

[6] J. Yu and P. Lu, "Learning traffic signal phase and timing information from low-sampling rate taxi GPS trajectories," Knowledge-Based Systems, vol. 110, no. 1, pp. 275-292, 2016.

[7] K. P. Wijayaratna, V. V Dixit, and L. Denant-Boemont, "An experimental study of the Online Information Paradox: does en-route information improve road network performance?" PLoS ONE, vol. 12, no. 9, pp. 184-191, 2017.

[8] Z. Wang, H. Ren, Q. Shen, W. Sui, and X. Zhang, "Seismic performance evaluation of a steel tubular bridge pier in a fivespan continuous girder bridge system," Structures, vol. 31, no. 1, pp. 909-920, 2021.

[9] S. Nakayama and J. Takayama, "Traffic network equilibrium model for uncertain demands," in Proceedings of the 82nd Transportation Research Board Annual Meeting, Washington, DC, USA, 2021.

[10] H. Shao, W. H. K. Lam, and M. L. Tam, "A reliability-based stochastic traffic assignment model for network with multiple user classes under uncertainty in demand," Networks and Spatial Economics, vol. 6, no. 3, pp. 173-204, 2019.

[11] A. Chen, J. Kim, and S. Lee, "Stochastic multi-objective models for network design problem[J]," Expert Systems with Applications, vol. 37, no. 2, pp. 1608-1619, 2020.

[12] H. Wang, W. H. K. Lam, X. Zhang, and H. Shao, "Sustainable transportation network design with stochastic demands and chance constraints," International Journal of Sustainable Transportation, vol. 9, no. 2, pp. 126-144, 2015.
[13] S.-M. Hosseininasab and S.-N. Shetab-Boushehri, "Integration of selecting and scheduling urban road construction projects as a time-dependent discrete network design problem," European Journal of Operational Research, vol. 246, no. 3, pp. 762-771, 2015.

[14] S.-M. Hosseininasab, S.-N. Shetab-Boushehri, S. R. Hejazi, and H. Karimi, "A multi-objective integrated model for selecting, scheduling, and budgeting road construction projects," European Journal of Operational Research, vol. 271, no. 1, pp. 262-277, 2018.

[15] M. Johnson, M. Schuster, Q. V. Le et al., “Google's multilingual neural machine translation system: enabling zero-shot translation," Transactions of the Association for Computational Linguistics, vol. 5, no. 1, pp. 339-351, 2017.

[16] M. D. Moreno, "Translation quality gained through the implementation of the iso en 17100:2015 and the usage of the blockchain," Babel, vol. 1, no. 2, pp. 1-9, 2020.

[17] X. Wang, X. Yu, L. Guo, F. Liu, and L. Xu, "Student performance prediction with short-term sequential campus behaviors," Information, vol. 11, no. 4, p. 101, 2020.

[18] Q. Guo, Z. Zhu, Q. Lu, D. Zhang, and W. Wu, "A dynamic emotional session generation model based on Seq2Seq and a dictionary-based attention mechanism," Applied Sciences, vol. 10, no. 6, pp. 1-10, 2020.

[19] H. Ren, Xi Mao, W. Ma, J. Wang, and L. Wang, “An EnglishChinese machine translation and evaluation method for geographical names," ISPRS International Journal of GeoInformation, vol. 9, no. 3, pp. 193-201, 2020.

[20] J. Arús-Pous, T. Blaschke, S. Ulander, J.-L. Reymond, H. Chen, and O. Engkvist, "Exploring the GDB-13 chemical space using deep generative models," Journal of Cheminformatics, vol. 11, no. 1, pp. 20-29, 2019.

[21] T. Moon, T. In Ahn, and E. Jung, "Long short-term memory for a model-free estimation of macronutrient ion concentrations of root-zone in closed-loop soilless cultures," Plant Methods, vol. 15, no. 1, pp. 1-12, 2019.

[22] N. Pourdamghani and K. Knight, "Neighbors helping the poor: improving low-resource machine translation using related languages," Machine Translation, vol. 33, no. 3, pp. 239-258, 2019.

[23] L. Bote-Curiel, S. Muñoz-Romero, A. Gerrero-Curieses, and J. Luis Rojo-Álvarez, "Deep learning and big data in healthcare: a double review for critical beginners," Applied Sciences, vol. 9, no. 11, pp. 1-11, 2019.

[24] J. Zhang and T. Matsumoto, "Corpus augmentation for neural machine translation with Chinese-Japanese parallel corpora," Applied Sciences, vol. 9, no. 10, pp. 1-12, 2019.

[25] Y. Chen, Y. Ma, X. Mao, and Q. Li, "Multi-task learning for abstractive and extractive summarization," Data Science and Engineering, vol. 4, no. 1, pp. 14-23, 2019.

[26] P. Zhou and Z. Jiang, "Self-organizing map neural network (SOM) downscaling method to simulate daily precipitation in the Yangtze and Huaihe River Basin," Climatic and Environmental Research, vol. 21, no. 5, pp. 512-524, 2016.

[27] X. Xiao, "Analysis on the employment psychological problems and adjustment of retired athletes in the process of career transformation," Modern Vocational Education, vol. 5, no. 12, pp. 216-217, 2018.

[28] S. Sahoo and M. K. Jha, "Pattern recognition in lithology classification: modeling using neural networks, self-organizing maps and genetic algorithms," Hydrogeology Journal, vol. 25, no. 2, pp. 311-330, 2016. 
[29] Y. Zhou and B. Yang, "Sports video athlete detection using convolutional neural network," Journal of Natural Science of Xiangtan University, vol. 39, no. 1, pp. 95-98, 2017.

[30] J. Pang, "Research on the evaluation model of sports training adaptation based on self-organizing neural network," Journal of Nanjing Institute of Physical Education, vol. 16, no. 1, pp. 74-77, 2017.

[31] G. Querzola, C. Lovati, C. Mariani, and L. Pantoni, "A semiquantitative sport-specific assessment of recurrent traumatic brain injury: the TraQ questionnaire and its application in American football," Neurological Sciences, vol. 40, no. 9, pp. 1909-1915, 2019.

[32] J. Wang, X. Luo, and H. Yan, "Correlation analysis between injuries and functional movement screening for athletes of the National Shooting Team," Journal of Capital Institute of Physical Education, vol. 5, no. 4, pp. 352-355, 2016. 